\title{
Multiple peer effects in the diffusion of innovations on social networks: a simulation study
}

\author{
Hang Xiong ${ }^{1 *}$ (D), Puqing Wang ${ }^{2}$ and Georgiy Bobashev ${ }^{3}$
}

\footnotetext{
*Correspondence: hxiong@ethz.ch ${ }^{1}$ Agricultural Economics and Policy Group, ETH Zurich, 8092 Zurich, Switzerland

Full list of author information is available at the end of the article
}

\begin{abstract}
Peer effects in innovation adoption decisions have been extensively studied. However, the underlying mechanisms of peer effects are generally not explicitly accounted for. Gaps in this knowledge could lead to misestimation of peer effects and inefficient interventions. This study examined the role of two mechanisms-sharing experiences (namely, experience effect) and externalities-in the adoption of an agricultural innovation. By referring to the diffusion process of a new crop in Chinese villages, we developed a simulation model that incorporated experience effect and externality effect on a multiplex network. The model allowed us to estimate the influence of each specific effect and to investigate the interplay of the positive and negative directions of the effects. The main results of simulation experiments were the following: (1) a negative externality effect in the system caused the diffusion of innovation to vary around a middle-level rate, which resulted in a fluctuating diffusion curve rather than a commonly found S-shaped one; (2) in the case of full diffusion, experience effect significantly shaped the diffusion process at the early stage, while externality effect mattered more at the late stage; and (3) network properties (i.e. connectivity, transitivity, and network distance) imposed indirect influence on diffusion through specific peer effects. Overall, our study illustrated the need to understand specific causal mechanisms when studying peer effects. Simulation methods such as agent-based modelling provide an effective approach to facilitate such understanding.
\end{abstract}

Keywords: Peer effects, Innovation diffusion, Social network, Agent-based simulation

\section{Background}

Social interactions can significantly shape individuals' economic behaviours. This is especially true when an individual behaves upon situations with uncertainties. In particular, an individual's decision on whether to adopt an innovation (i.e. the idea, practice, or object that are perceived as new (Rogers 2003)) often depends on the decisions of their friends, relatives, colleagues, etc. Such social influence is referred to as peer effects. Existing studies have examined peer effects on the diffusion of innovations in varied settings, including products and services (Goolsbee and Klenow 1999; Sorensen 2006; Kremer and Levy 2008; Luan and Neslin 2009), technologies (Munshi 2004; Bandiera and Rasul 2006; Conley and Udry 2010), financial services (Banerjee et al. 2013), and social programmes (Dahl et al. 2014). In these studies, many forms of peer effects, including word of mouth (Luan and Neslin 2009; Banerjee et al. 2013), social learning (Bandiera and Rasul 2006;

(c) The Author(s). 2018 Open Access This article is distributed under the terms of the Creative Commons Attribution 4.0 International License (http://creativecommons.org/licenses/by/4.0/), which permits unrestricted use, distribution, and reproduction in any medium, provided you give appropriate credit to the original author(s) and the source, provide a link to the Creative Commons license, and indicate if changes were made. 
Conley and Udry 2010), and network externalities (Goolsbee and Klenow 1999), are discussed ${ }^{1}$.

The work by (Xiong et al. 2016) distinguishes three basic interactions through which peer effects take place in the diffusion of innovations: transmitting information, sharing experiences and externalities. They are termed as information effect, experience effect, and externality effect, respectively. Specifically, information effect refers to the influence of the transmission of awareness information of the innovation and general information about the cost and benefit of adopting the innovation. The effect can occur through any relationship ties through which individuals can communicate. Experience effect characterises the influence that one obtains by sharing experiential knowledge (e.g. know-how, localised techniques) or physical resources (e.g. seeds of a new crop, tools) from earlier adopters. Such knowledge and resource are generally scarce at this stage of the diffusion process. Experience effect thus mainly occurs through close social relationships, such as kinship or close friendship. In addition, an individual's adoption behaviour can lead to positive or negative externalities. They can affect other individuals regardless of whether those individuals also adopt the innovation. Negative externalities leads to the reduction of payoff when staying on the original choice, and consequently increases individuals' propensity to choosing the innovation, which in turn increases the diffusion in the group, generating a positive externality effect. Likewise, positive externalities can generate a negative externality effect. In empirical studies, negative externality effect is rarely considered mainly due to the difficulty of collecting data. In general, the three effects have significant impacts on different stages of the diffusion process. Information effect shapes the process mainly at the early stage, experience effect at the intermediate stage, and externality effect at the late stage as depicted in Fig. 1.

Peer effects were previously studied either as a composite of different mechanisms or in a specific form (such as social leaning or network externalities). Typically only one mechanism was considered. However, a diffusion process is very often shaped by multiple mechanisms simultaneously, and each mechanism could play a different role at a different phase of the evolution of the process. Different mechanisms may potentially have different policy implications (Carrell et al. 2013; Alcalde 2013). This paper is a theoretical study to examine the roles of multiple peer effects

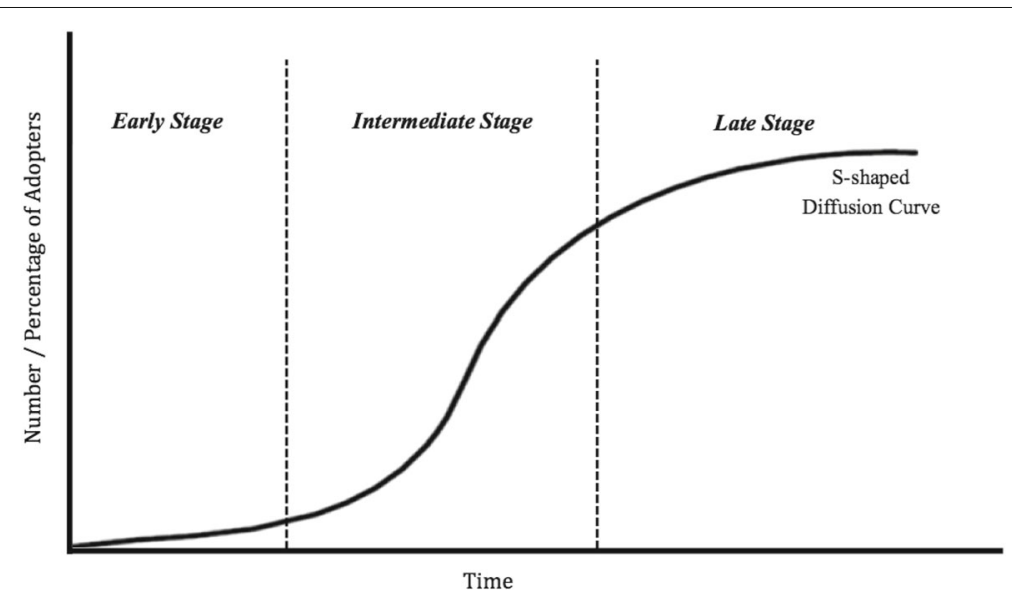

Fig. 1 Peer effects at different stages of the diffusion process 
in the diffusion of innovations. We have chosen a case of the adoption of a new crop in rural China to set up our simulation model and to select key experimental parameters. In this case, all households were well informed of the new crop at the beginning of the diffusion, and thus, information effect is excluded from our model. Our model and estimation are therefore focused on experience effect and externality effect.

\section{Background and data}

This paper use the case of the diffusion of a high-value crop (a crop with high economic return) in 10 villages in rural China to demonstrate multiple types of peer effects in the adoption of an innovation. The farmers in these villages traditionally farmed staple rice and cotton. The new vegetable, Artemisia selengensis (AS), was introduced into the villages in 2001. To encourage farmers to grow AS, village leaders distributed some seed-stalks to each household for free. This action informed all households about the new crop. However, as the farmers were not certain about the profitability of farming a new crop, there were only one or two households in each village (the average number of households was 37, with a standard deviation of 14) who adopted in the first year. These earlier adopters were mainly motivated by the awareness information they obtained. Their adoption behaviour was thus the result of the information effect.

When other farmers realised that farming the AS was much more profitable, they also started to farm in subsequent years. However, they encountered difficulty in obtaining seed-stalks, which can only remain fresh for a few days. It turned out that the only reliable source at the time was the earlier adopters in their villages. The seed-stalks were so scarce at the time that a household could obtain them exclusively from its close relatives. Those who managed to obtain the seed-stalks in a given year then shared them with other households next year, and so forth. Meanwhile, new adopters were often benefited by obtaining techniques and tacit knowledge from their relatives and house neighbours who have adopted earlier. Overall, the sharing of such experiential resources provided the main motivation for farmers' adoption in this stage. By 2005 , more than $70 \%$ of the households had adopted AS.

When the majority of the households by then had adopted, the non-adopters were put under some pressure to join in, mainly through the use of irrigation. There was nearly a half-year period during which both AS and cotton were active on the land. However, farming AS required much more water than farming cotton. A plot for farming cotton could thus be over flooded as its adjacent plots were planted with AS. Therefore, households farming cotton in this situation would be 'coerced' into adopting AS. Such adoption was thus attributed to positive externality effect.

Figure 2 presents adoption rates of the new crop throughout the entire diffusion period. It shows an S-shaped diffusion curve as many other studies do. This case provides an excellent demonstration for the study of different types of peer effects in a complete diffusion process.

The data collected from this case are used to calibrate the parameters in the model. They include the number of households (i.e. nodes in the social network), characteristics of household (e.g. risk preference) ratio of initial adopters (elaborated in the "Model parameterisation" section). 


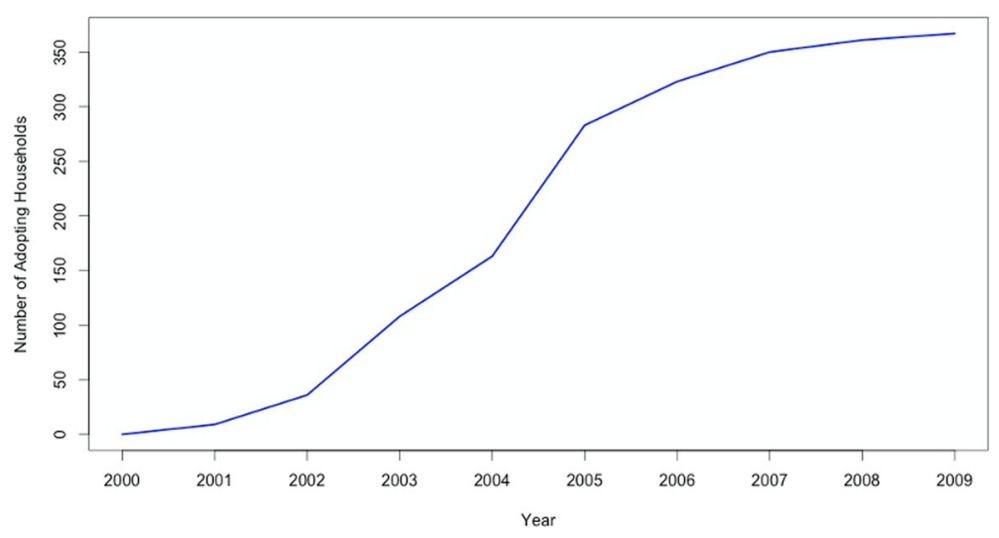

Fig. 2 Diffusion of the new crop in the Chinese villages

\section{Methods and model}

In this section, we first justify our choice of agent-based modelling for conducting this study. We then described how agents' behavioural environment (i.e. the social networks) and agents' decision-making are represented in our model. Finally, we present how parameters in the model are set up.

\section{Agent-based modelling approach}

The underlying mechanisms following which peer effects occur in a diffusion process are generally nuanced and subtle. Empirical analysis is largely restricted because of the difficulty of data collection. Moreover, traditional approaches such as regression analysis are not capable to explicitly represent dynamic behavioural mechanisms of such. Additionally, negative peer effects in diffusions seldom enter researchers' notice. This is because failed or incomplete diffusions, which negative effects often end up with, do not draw much attention. In this study, we conducted a theoretical study following the agent-based modelling approach. This approach is able to incorporate micro-level adoption mechanisms, individuals' heterogeneity, and social relationships among individuals (Kiesling et al. 2012). Particularly for this study, it allows for the incorporation of the negative externality effect. This approach has been used to examine, for instance, the negative effects of word of mouth (Goldenberg et al. 2001; Deffuant et al. 2005).

We developed an agent-based model with peer interactions being structured in a multiplex network, that is, a network consisting of multiple layers, on each of which a type of relationship is mapped (Arenas et al. 2014). This approach allows us to identify different peer effects in the system (Bramoulle et al. 2009; Goldsmith-Pinkham and Imbens 2013). To keep the model simple, we only include two effects: experience and externality. The development of our model is based on two assumptions.

Assumption 1: Peer effects exist and impose impacts on the diffusion process in the social system in study.

This assumption is made based on the theoretical analysis above as well as the observations from the real world. It permits us to circumvent the quagmire of whether peers affect each other and be focusing on the discussion on how this influence occurs (Guryan et al. 2008). This assumption is the prerequisite of to the simulation of the impact of peer effects, though it is often not explicitly stated in literature. 
Assumption 2: Different types of specific peer effect (mainly) occur through different types of social relationship.

In our study, specific peer effects are distinguished according to the kind of social interactions through which they take place. It is observed that a particular type of social interaction tends to mainly take place through a particular type of social relationship (or a particular set of different types). Therefore, it is plausible that researchers are able to specify through which type(s) of relationship different specific peer effect occur in a system. This assumption enables us to model different specific peer effects on different network layers and examine how the characteristics of a network layer shape the peer effect on that layer.

\section{Setup of network structure}

We took the real-world case described in the "Background and data" section as a reference to design the simulation. We modelled the diffusion of the new crop using an agent-based model. In the model, the social system (environment) is village, represented as a social network. The agents were the households in the village, represented as nodes on the social network. In the villages, the interactions among households that substantially shape the diffusion mainly occurred through kinship ties, house neighbourhood ties and land plot neighbourhood ties ${ }^{2}$.

In the model, a small portion of households are set to have adopted the innovation in the initial time. They can be considered as those adopted due to the awareness of the innovation. In other words, our model ignores information effect and focuses on experience effect and externality effect. According to the previous discussion, we reasonably assumed that experience effect occurs through kinship ties and house neighbourhood ties, whereas externality effect occurs through land plot neighbourhood ties. Since the kinship ties and house neighbourhood ties are highly overlapped in these villages (as the land for building houses were to large extent allocated based on extended family, house neighbours are often family members), we term the network consists of the two types of ties as kinship network for the sake of simplicity. The network consists of land plot neighbourhood ties is thus termed as neighbourhood network. The social network on which the peer effects occur are structured thus has two layers: the kinship network layer and the neighbourhood network layer, as demonstrated in Fig. 3.

We then simulated the kinship network as a Watts-Strogatz (WS) small-world network (Watts and Strogatz 1998) and simulated the neighbourhood network as an Erdös-Rényi (ER) random network (Erdős and Rényi 1959). These settings are reasonable. First, the kinship networks in these villages are very similar to small-world networks according the empirical study (Xiong and Payne 2017) and this finding echoes the classic theory of 'acquaintance society' on the structure of traditional Chinese structure. Second, the land plot neighbourhood networks are close to random networks according to the same empirical study. This is the case because the plots were originally distributed by lot. The parameters defining the network structures were roughly calibrated according to the network characteristics of the villages in our real-world case.

\section{Simulation of agents' decision-making}

Two approaches are widely utilised to describe diffusion in social groups. One is a probability-based approach (e.g. (Banerjee et al. 2013; Peres 2014)), where individuals' 


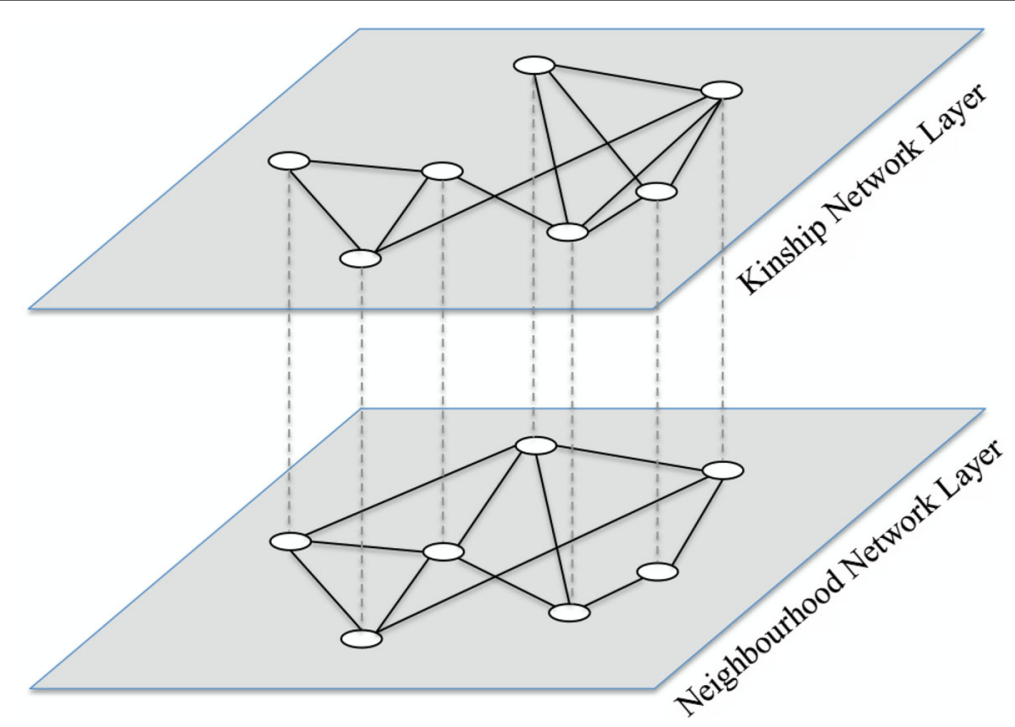

Fig. 3 A two-layer multiplex network

probability of adopting the innovation increases as the fraction of peers that adopt increase. The other is a threshold-based approach (e.g. (Granovetter 1978; Singh et al. 2013)), where individuals change their adoption state when a certain threshold of utility is reached. In the case of this study, on the kinship network, the more an individual's peers have adopted the new crop, the more likely that the individual gains information and thus follow suit. We therefore simulated experience effect on the kinship network following the probability-based approach. The probability for an individual to adopt is proportional to the fraction of his family members who have adopted. Meanwhile, the externalities of planting the new crop are mainly determined by the nature of the crop itself, such as its compatibility with the existing crop. This can be viewed as a threshold that all practitioners face. Once the fraction of one's neighbours who adopt reaches the threshold, an individual will adopt for sure. Thus, the externality effect occurring on the neighbourhood network is modelled using a threshold.

Suppose there are $N$ nodes in the network, i.e. on each of the two layers. On the kinship network, node (household) $i$ chooses to adopt the innovation with a probability $p_{i}$. The probability is determined by the fraction of his adopting peers $f_{i}^{\text {kin }}$ as well as his risk preference (a representative of the individual's personal characteristics) $x_{i} \in(0,1)$. Therefore, $p_{i}=\theta f_{i}^{\mathrm{kin}} x_{i}$, where $\theta \in(0,1)$ is a coefficient indicating the degree of information effect (namely information coefficient). On the neighbourhood network, household $i$ changes his adoption state when the fractions of his adopting neighbours $f_{i}^{\text {geo }}$ reaches a unified adoption threshold (namely externality threshold, denoted by $\delta$ ), which is determined by the characteristics of the new crop. When the threshold is reached, in the scenario of the positive externality effect, the non-adopters switch to adopting state; in the scenario of negative externality effect, the adopters change back to non-adopting.

A household has two adoption status: adopting or non-adopting. We track households' adoption states and the ratio of adopting households (namely the adoption rate) at the village level. The basic algorithm of the simulation is as follows:

(i) Initial time $t=0$ 
A fraction $\lambda$ of seed adopters is selected from the population of the households at random. Meanwhile, their adoption states are set to be adopting.

(ii) Iteration at time $t$,

Households update their state through kinship ties and neighbourhood ties simultaneously.

(a) Updating on the kinship network

The non-adopters adopt with probability $p_{i t}=\theta f_{i t}^{\mathrm{kin}} x_{i}$.

(b) Updating on the neighbourhood network

In the scenario of positive externality effect, non-adopting household $i$

transmits to adopting state once the fraction of his adopting neighbours

reaches the externality threshold, i.e. $f_{i t}^{\text {geo }}>\delta$.

In the case of negative externality effect, adopting household $i$ transmits to non-adopting state once the fraction of his adopting neighbours reaches the externality threshold, i.e. $f_{i t}^{\text {geo }}>\delta$.

The two updating mechanisms occur simultaneously in reality, so we set the order to be random when updating on the two layers in the model. The process repeats until any of the following termination rules is satisfied: (i) the adoption rate is lower than $5 \%$ (which is the lowest ratio of seed adopters), (ii) the adoption rate is higher than $95 \%$ (i.e. complete diffusion), and (iii) it iterates for 20 times - this is sufficient for the system to converge in most cases (both in the simulation and in the real world).

\section{Model parameterisation}

The parameters in the simulation model are set using the survey data from the 10 reference villages. Parameter values are set to the mean estimates from the data. The parameters were set up as follows:

- Number of nodes (the population) $N \in[20: 20: 80]$

- Fraction of rewiring nodes in the generation of WS small-world networks $W S_{f} \in\{0.05,0 . s, 0.15\}$

- Rewiring probability in the generation of ER random networks $\mathrm{ER}_{\text {prob }} \in\{0.10,0.15,0.20\}$

- Experience coefficient $\theta \in[0.05: 0.05: 0.25]$

- Externality threshold $\delta \in[0.6: 0.05: 0.80]$

- Ratio of seed adopters $s \in[0.05: 0.05: 0.20]$

- Risk preference of household $i, x_{i}$. The value is generated as a positive random number with normal distribution (mean 1 and standard deviation 0.3 ; truncated to 0 and 2 for values less than 0 and greater than 2 respectively).

We run each parameter combination 100 times. There are 3600 (full factorial) combinations in total, and the model runs each of these combinations 100 times. Finally, 360,000 sets of simulation results were generated.

\section{Results and discussion}

Convergence adoption rates and diffusion curves

Scenario of positive externality effect

Convergence adoption rates. The densities of convergence adoption rates and convergence rounds are presented in Fig. 4. The left panel shows that approximately $85 \%$ of the 
runs converge to full adoption (that is, as we defined, more than $90 \%$ of the population have adopted) within 20 iterations. It means that most individuals adopt eventually. The right panel indicates how many rounds it takes for the runs to converge. More than $40 \%$ of the runs converge in five rounds. The density decreases with the number of rounds ${ }^{3}$. In this scenario, both the two diffusion mechanisms are positive feedback mechanisms, so it is very likely that the system will converge to complete diffusion.

S-shaped diffusion curves. The S-shaped adoption curve is a typical pattern of the diffusion. It holds because most innovations bear the following feature: The adoption rate grows slowly at the beginning of the diffusion process. When the diffusion reaches the critical mass, it will have a sharp increase. After that, it will slowly approach complete diffusion. The variance lies in the slope of the curve. The innovations diffusing rapidly generate steep curves, whereas those diffusing slowly generate flat curves. Our simulations successfully generate $\mathrm{S}$-shaped diffusion curves. Figure 5 displays the curves for the diffusions converge at the sixth round, and the ratio of seed adopters is 0.05 and 0.1 , respectively ${ }^{4}$.

\section{Scenario of negative externality effect}

Convergence adoption rates. The diffusion converges to diverse adoption rates in this scenario. As shown in Fig. 6, nearly 70\% of the simulations converge to an adoption rates between 40 and $80 \%$. Specifically, more than $25 \%$ converge to a value in the interval of $50-60 \%$ and more than $25 \%$ converge to a value between 60 and $70 \%$. Only about $5 \%$ end up with full adoption. More than $90 \%$ of the simulations converged within 20 rounds.

When a negative effect is introduced, the diffusion curve dramatically changes its shape. Converging to full adoption is not a certain outcome any more. At which adoption rate the diffusion will converge depends on the competition between the positive effect and the negative effect. This result provides an interpretation of the incomplete diffusion phenomena in the real world. For instance, in the case of mircofinance (Banerjee et al. 2013), the average take-up rate is $18.5 \%$ over all rural communities that were studied. Most innovations end up with not been taken up by all potential adopters in the social group. New fashionable clothes can be a typical example. When they go from high fashion to street
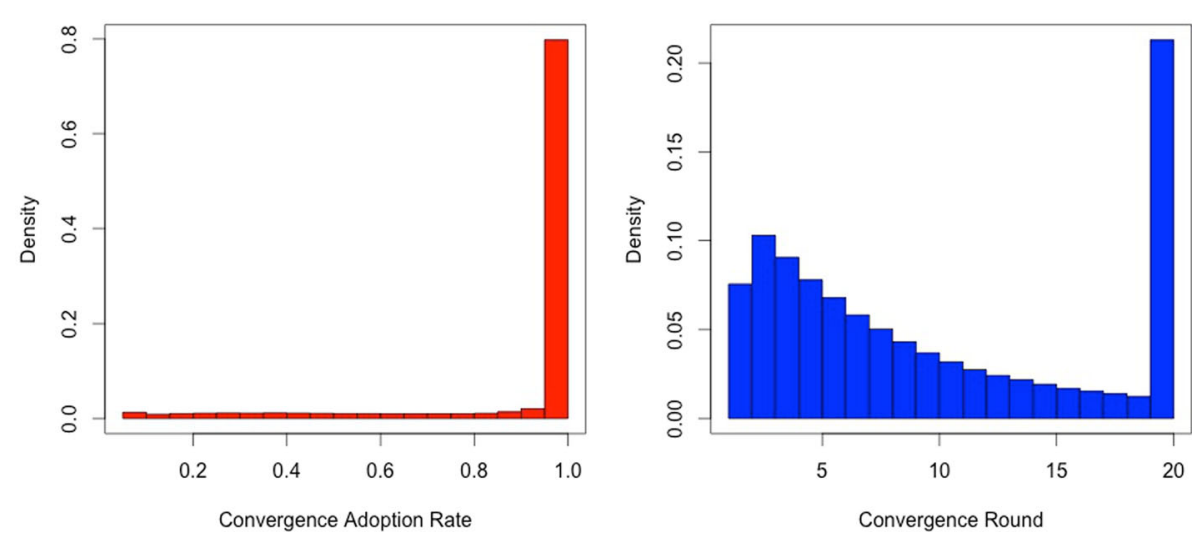

Fig. 4 Histograms of convergence adoption rates and convergence rounds (positive externality effect) 


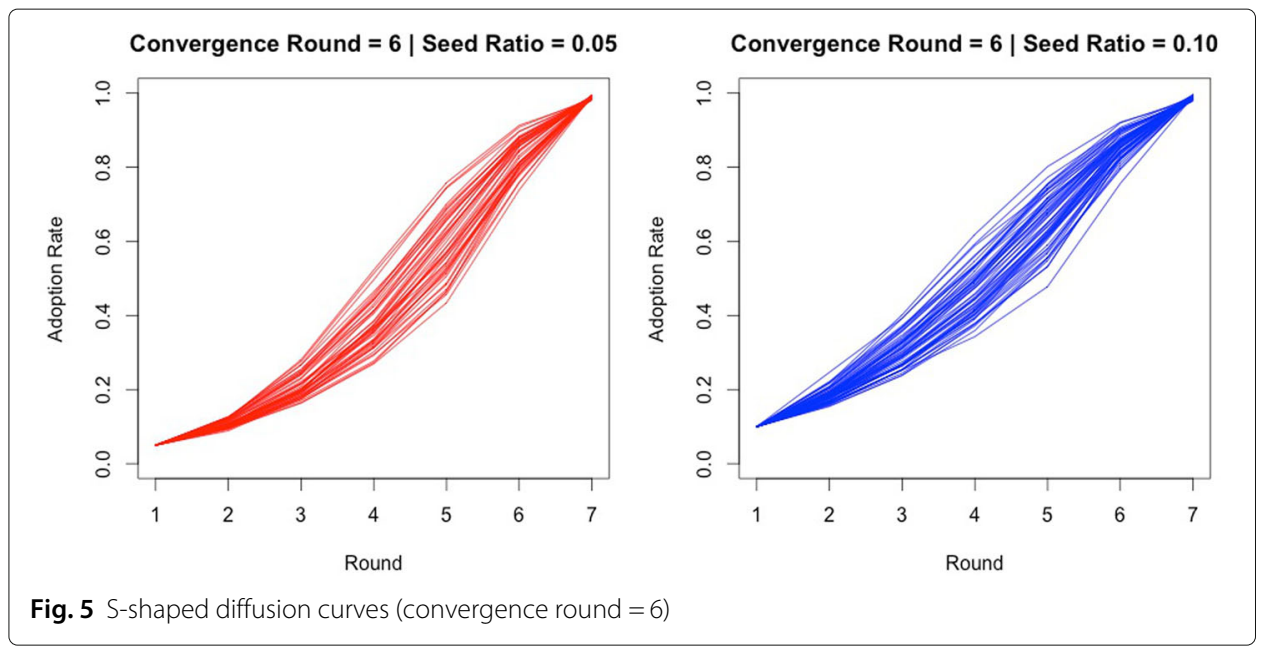

fashion, they are not considered fashionable any more, and thus become less attractive. This negative effect leads the diffusion to converge before reaching full adoption.

Fluctuating diffusion curves. Figure 7 displays the diffusion curves for the simulations that converge, again, at the sixth round with the ratios of seed adopters 0.05 and 0.1 , respectively. To exhibit the trends of the curves, the adoption rates after the convergence are also plotted. The graph shows that the diffusion curves fluctuate around a value in the middle of 0 and 1 (approximately 0.6 in our case). The specific convergence value depends on the relative strength of the two opposite effects in the diffusion process.

Overall, when all specific diffusion mechanisms in the system are in favour of diffusion, the system will almost always converge to complete diffusion, and an S-shaped diffusion curve will be generated. When there is a negative mechanism, it reshapes the diffusion to fluctuate around a middle-level rate, hence generating fluctuating diffusion curves.

\section{Experience effect and externality effect}

We explore how experience effect and externality effect influence the effectiveness of diffusion, which is measured by the reach of the diffusion and its speed. The two measures are both related to adoption rate, the fraction of households that have adopted at a round
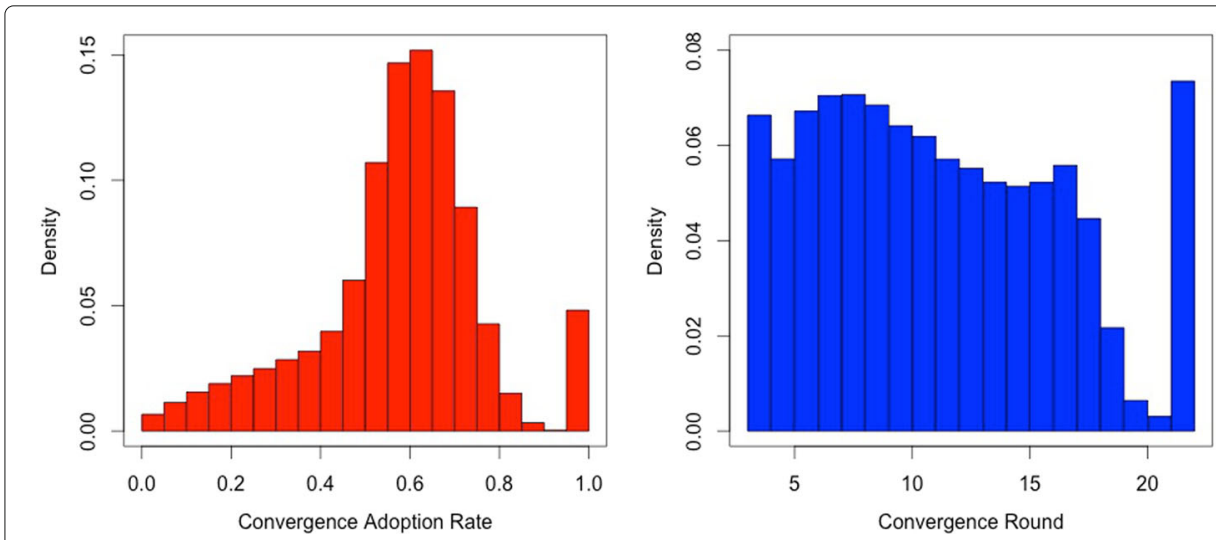

Fig. 6 Histograms of convergence adoption rates and convergence rounds (negative externality effect) 


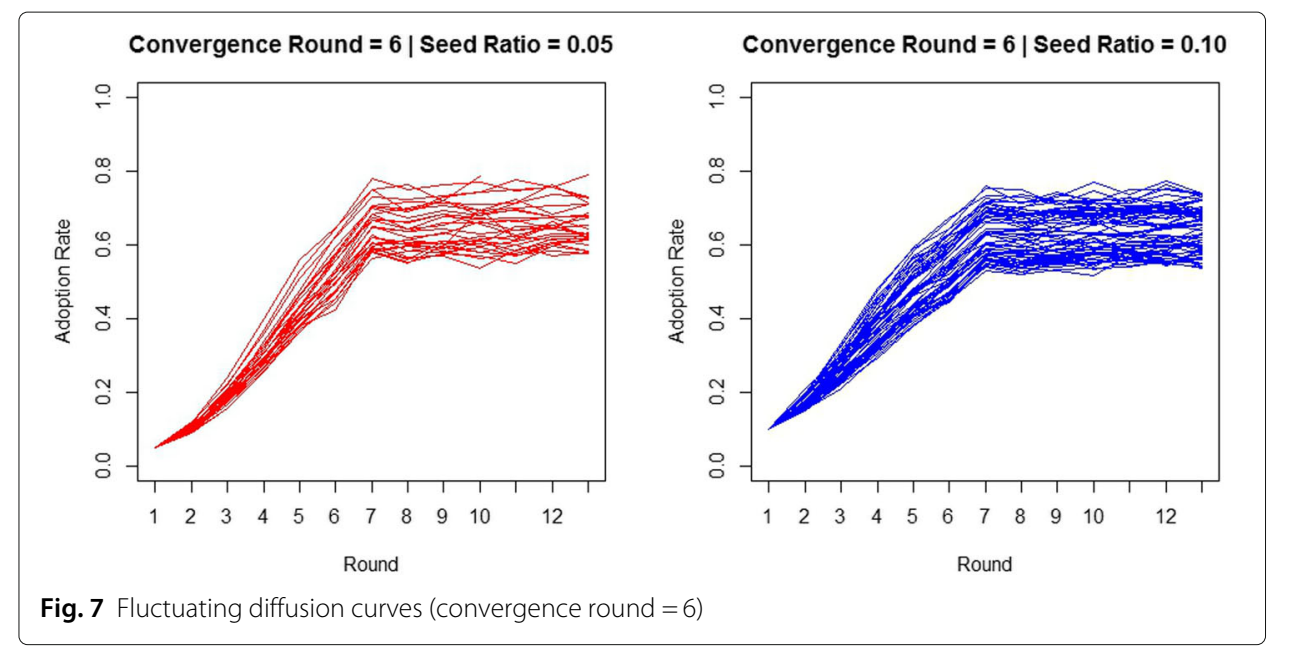

to the whole population. Specifically, convergence adoption rate, reflecting the reach of the diffusion, is defined as the adoption rate at which the diffusion converges. Diffusion speed is defined as the increase of the adoption rate per round, or namely, the expression of the adoption rate achieved by the number of rounds to achieve it ${ }^{5}$. However, the two metrics cannot be both used in positive and negative externality effect at the same time. When the externality effect is positive, the diffusion almost always converges to $100 \%$, so the convergence adoption rate is not a feasible metric. Likewise, the diffusion speed does not apply when the externality effect is negative because the adoption rate oscillates over the diffusion process, but we can use convergence speed (how fast the diffusion process converges) as a substitute. It is the quotient of convergence adoption rate by the number of rounds it takes to achieve the rate. Therefore, the diffusion speed is used in the scenario of positive externality effect, whereas the convergence adoption rate and convergence speed are employed in the scenario of negative externality effect.

In the model, the experience effect and the externality effect are reflected by the experience coefficient and the externality threshold, respectively. Higher experience coefficient indicates higher experience effect, whereas higher externality threshold indicates lower externality effect. To estimate their impact on the effectiveness of diffusion, we run a multivariate regression using the following equation:

$$
y=\alpha_{0}+\alpha_{1} \theta+\alpha_{2} \delta+\alpha_{3} s+W^{\prime} \alpha_{4}+\epsilon
$$

where $y$ is the diffusion speed (in the scenario of positive externality effect) or the convergence adoption rate (in the scenario of negative externality effect), $\theta$ is the experience coefficient, $\delta$ is the externality threshold, $s$ is the ratio of initial seed adopters, and $W^{\prime}$ is the network characteristic controls, including number of nodes $N$, parameters used in generating ER network (i.e. rewiring probability $\mathrm{ER}_{\mathrm{prob}}$ ), and WS network (i.e. fraction of rewiring nodes $W S_{f}$ ).

We note that the traditional null hypothesis test is not well suited for simulation studies because with a sufficient number of simulated trajectories one can make the $p$ value arbitrarily small (Heard et al. 2014). Thus, we conduct a traditional minimum-effect test. We test that the difference is bigger than an a priori defined minimal meaningful effect (MME). Parameter values significantly smaller than the MME signify no effect. Parameter 
values significantly large than MME signify significant difference, and when the statistical significance is not achieved, it signifies that no conclusion could be made given the data. Under such paradigm, the increase in the number of simulations will increase the precision and reduce the $p$ values but will not produce artificially significant small-effect sizes (Wellek 2010). Such approach is also the basis for a classic power analysis, which requires to explicitly define a minimally meaningful effect size. In our study, we have selected the MME to be 0.1 standard deviation. Our judgement on the significance of estimated values in the regression results in this study are based on the minimum-effect test (whether they are significantly larger than the minimal effect defined as 0.1 of the standard deviation).

\section{Scenario of positive externality effect}

We regress the diffusion speed when the adoption rate reaches $30 \%, 40 \%$, up to $90 \%$. Table 1 presents the results ${ }^{6}$. First, all the estimated values were significantly larger than the minimal effect defined as 0.1 of the standard deviation. Specifically, both the experience coefficient and the externality threshold in the simulation are highly significantly associated with the diffusion speeds, and the signs are in accordance with expectations. This indicates that they both impose a significant influence on the diffusion. More importantly, we found the influence of the experience effect wanes as the adoption rate increases (i.e. the diffusion process), while the influence of the externality effect continuously grows. At the earlier stage, the adopters are few, so only a small number of households can reach the externality thresholds. Consequently, the externality effect has a limited impact on the diffusion. Meanwhile, the experience effect, which is continuous and steady, has a major contribution. At the later stage, there are more adopters; thus, more households can reach the externality threshold. Externality effect, therefore, become relatively higher. However, this could undermine the influence of experience effect, because the two effects can substitute each other-a household can make the adoption decision because of either experience effect or externality effect. Such a substitution effect becomes larger as the adoption rate increases.

\section{Scenario of negative externality effect}

We run the regression also using Eq. (1). The same explanatory variables as in the scenario of positive externality effect (the experience coefficient, the externality threshold, the ratio

Table 1 Regressions of adoption speed at different diffusion levels (positive externality effect)

\begin{tabular}{llllllll}
\hline & $\begin{array}{l}\text { Model 1 } \\
(30 \%)^{*}\end{array}$ & $\begin{array}{l}\text { Model 2 } \\
(40 \%)\end{array}$ & $\begin{array}{l}\text { Model 3 } \\
(50 \%)\end{array}$ & $\begin{array}{l}\text { Model 4 } \\
(60 \%)\end{array}$ & $\begin{array}{l}\text { Model 5 } \\
(70 \%)\end{array}$ & $\begin{array}{l}\text { Model 6 } \\
(80 \%)\end{array}$ & $\begin{array}{l}\text { Model 7 } \\
(90 \%)\end{array}$ \\
\hline (Intercept) & -0.53 & -0.50 & -0.47 & -0.45 & -0.43 & -0.32 & -0.32 \\
Number of households & 0.01 & 0.01 & 0.01 & 0.01 & 0.01 & 0.01 & 0.01 \\
Ratio of seed adopters & 0.90 & 0.84 & 0.81 & 0.80 & 0.78 & 0.57 & 0.57 \\
Experience coefficient & 2.04 & 2.02 & 2.01 & 1.99 & 1.94 & 1.54 & 1.54 \\
Externality threshold & -0.18 & -0.21 & -0.23 & -0.25 & -0.26 & -0.28 & -0.28 \\
\% of neighbours to connect in WS & 3.23 & 3.24 & 3.23 & 3.21 & 3.16 & 2.53 & 2.53 \\
Rewiring probability in ER & -0.15 & -0.14 & -0.12 & -0.10 & -0.08 & 0.09 & 0.09 \\
$R^{2}$ & 0.75 & 0.78 & 0.80 & 0.80 & 0.80 & 0.72 & 0.72 \\
\hline *The adoption rate level for diffusion speed in the present regression. This is the same for Tables 4, 5, and 6 & & &
\end{tabular}


of initial seed adopters, and the network characteristics) are employed. They are regressed against two response variables, the convergence adoption rate (model 1 in Table 2) and the convergence speed (model 2 in Table 2). The results show that both the experience effect and the externality effect are significantly correlated to these response variables with expected signs, suggesting that they both have significant impacts on diffusion ${ }^{7}$.

\section{Network characteristics and peer effects}

The diffusion of innovation is usually influenced by the structure of the network in which it takes place (Peres 2014). We measure the structure of the networks used in our simulation using three metrics: average degree, clustering coefficient, and average path length. They are chosen because they reflect different aspects of the characteristics of a network: average degree indicates how connected the network is (i.e. the connectivity); clustering coefficient measures how clustered the nodes are and thus how well the information can transmit (i.e. the transitivity); and average path length represents how many intermediaries it needs for one node to reach another (i.e. the network distance).

To estimate the impact of these network topological metrics on the corresponding network layer, we introduce a interaction term into the regressions. The interaction term is generated by multiplying the experience coefficient or the externality threshold by each of the topological metrics on the corresponding network layer. The interaction effect can be interpreted from the regression coefficients of the interaction terms. As different topological metrics and the network generation parameters are highly correlated with each other (as presented in Table 3 for the scenario of positive externality effect, and those for the scenario of negative externality effect are similar), we run the regression for each topological metric separately (and drop the network generation parameters served as controls).

The resultant regression equation is the following:

$$
y=\beta_{0}+\beta_{1} \theta+\beta_{2} \delta+\beta_{3} \text { Net_char }+\beta_{4} \text { Inter_term }+\beta_{5} s+\epsilon
$$

where Net_char denotes the vector of topological metrics on the kinship network and the neighbourhood network, respectively, and Inter_term denotes the vector of interaction terms. The interaction term is the product of a topological metric and the corresponding peer effect indicator occurring on the network. For instance, the interaction term of average degree and peer effect on kinship network is (average degree of kinship network) $\times \theta$. One topological metric is run for each regression. As there are two scenarios, the regression runs in a total of 6 times $(3 \times 2)$.

Table 2 Regressions of convergence adoption rate and convergence speed (negative externality effect)

\begin{tabular}{lll}
\hline & $\begin{array}{l}\text { Model 1 } \\
\text { (Conv. Adp. rate) }\end{array}$ & $\begin{array}{l}\text { Model 2 } \\
\text { (Conv. speed) }\end{array}$ \\
\hline (Intercept) & 0.90 & -0.31 \\
Number of households & 0.00 & 0.01 \\
Ratio of seed adopters & 0.17 & 0.57 \\
Experience coefficient & 0.19 & 1.53 \\
Externality threshold & -0.05 & -0.28 \\
\% of neighbours to connect in WS & 0.39 & 2.53 \\
Rewiring probability in ER & -0.02 & 0.06 \\
$R^{2}$ & 0.11 & 0.72 \\
\hline
\end{tabular}


Table 3 Correlations between network characteristics (positive externality effect)

\begin{tabular}{lllllllll}
\hline & $(\operatorname{Var} 1)$ & $(\operatorname{Var} 2)$ & $(\operatorname{Var} 3)$ & $(\operatorname{Var} 4)$ & $(\operatorname{Var} 5)$ & $(\operatorname{Var} 6)$ & $(\operatorname{Var} 7)$ & $(\operatorname{Var} 8)$ \\
\hline WS & 1 & & & & & & & \\
Avg. Dg. of Kin. & 0.65 & 1 & & & & & \\
Clu. Coef. of Kin. & 0.83 & 0.69 & 1 & & & & \\
APL of Kin. & -0.73 & -0.64 & -0.86 & 1 & & & & \\
ER prob & & & & & 1 & & & \\
Avg. Dg. of Nei. & & & & & 0.49 & 1 & 1 & \\
Clu. Coef. of Nei. & & & & 0.71 & 0.43 & 1 \\
APL of Nei. & & & & -0.65 & -0.76 & -0.58 & 1 \\
\hline
\end{tabular}

\section{Scenario of positive externality effect}

As discussed previously, we use the diffusion speed as the response variable in the regressions. Results for average degree, clustering coefficient, and average path length are displayed in Tables 4, 5, and 6, respectively.

First, the coefficients of all the interaction terms with the three metrics are significant, indicating that interaction effects do exist. Specifically, average degree significantly enhances both the experience effect and the externality effect, that is, with the increase of average degree, their impacts on diffusion speed grow. As the system proceeds to a higher diffusion level, the interaction effect on the experience effect shrinks, while that on the externality effect grows. The degree of clustering also strengthens these two effects, although the influence is not significant for the externality effect when the diffusion is at the early stage. As the overall adoption rate grows, it turns significant and becomes stronger, while the influence for the experience effect falls gradually. The distance between households also significantly affects the performance of the two effects, but in an opposite direction (i.e. a lower distance corresponds to a higher experience effect or externality effect and vice versa). Comparing the three metrics, the clustering coefficient produces a much higher impact (while it is significant) than the others, whereas the average degree and the average path length have a weak influence. In other words, the transitivity of the network matters more than the connectivity and the distance in shaping peer effects.

Table 4 Regressions of adoption speed on average degree (positive externality effect)

\begin{tabular}{|c|c|c|c|c|c|c|c|}
\hline & $\begin{array}{l}\text { Model } 1 \\
(30 \%)\end{array}$ & $\begin{array}{l}\text { Model } 2 \\
(40 \%)\end{array}$ & $\begin{array}{l}\text { Model } 3 \\
(50 \%)\end{array}$ & $\begin{array}{l}\text { Model } 4 \\
(60 \%)\end{array}$ & $\begin{array}{l}\text { Model } 5 \\
(70 \%)\end{array}$ & $\begin{array}{l}\text { Model } 6 \\
(80 \%)\end{array}$ & $\begin{array}{l}\text { Model } 7 \\
(90 \%)\end{array}$ \\
\hline (Intercept) & -0.07 & -0.05 & -0.04 & -0.03 & -0.01 & 0.02 & 0.02 \\
\hline Number of households & -0.00 & 0.00 & 0.00 & 0.00 & 0.00 & 0.00 & 0.00 \\
\hline Ratio of seed adopters & 0.90 & 0.84 & 0.81 & 0.80 & 0.78 & 0.57 & 0.57 \\
\hline Experience coefficient (Var 1) & 0.87 & 0.99 & 1.07 & 1.08 & 1.02 & 0.74 & 0.74 \\
\hline Externality threshold (Var 2) & -0.16 & -0.19 & -0.21 & -0.23 & -0.23 & -0.22 & -0.22 \\
\hline Average degree of Kin. (Var 3) & 0.01 & 0.01 & 0.02 & 0.02 & 0.01 & 0.01 & 0.01 \\
\hline Average degree of Nei. (Var 4) & -0.00 & 0.00 & 0.00 & 0.00 & 0.00 & 0.01 & 0.01 \\
\hline $\operatorname{Var} 1 \times \operatorname{Var} 3$ & 0.12 & 0.10 & 0.10 & 0.09 & 0.09 & 0.08 & 0.08 \\
\hline $\operatorname{Var} 2 \times \operatorname{Var} 4$ & -0.01 & -0.00 & -0.00 & -0.00 & -0.00 & -0.01 & -0.01 \\
\hline$R^{2}$ & 0.80 & 0.81 & 0.82 & 0.82 & 0.82 & 0.75 & 0.75 \\
\hline
\end{tabular}


Table 5 Regressions of adoption speed on clustering coefficient (positive externality effect)

\begin{tabular}{llllllll}
\hline & $\begin{array}{l}\text { Model 1 } \\
(30 \%)\end{array}$ & $\begin{array}{l}\text { Model 2 } \\
(40 \%)\end{array}$ & $\begin{array}{l}\text { Model 3 } \\
(50 \%)\end{array}$ & $\begin{array}{l}\text { Model 4 } \\
(60 \%)\end{array}$ & $\begin{array}{l}\text { Model 5 } \\
(70 \%)\end{array}$ & $\begin{array}{l}\text { Model 6 } \\
(80 \%)\end{array}$ & $\begin{array}{l}\text { Model 7 } \\
(90 \%)\end{array}$ \\
\hline (Intercept) & -0.17 & -0.17 & -0.17 & -0.16 & -0.15 & -0.08 & -0.08 \\
Number of households & 0.00 & 0.00 & 0.00 & 0.00 & 0.00 & 0.00 & 0.00 \\
Ratio of seed adopters & 0.91 & 0.84 & 0.81 & 0.80 & 0.78 & 0.57 & 0.57 \\
Experience coefficient (Var 1) & 0.21 & 0.35 & 0.43 & 0.45 & 0.41 & 0.31 & 0.31 \\
Externality threshold (Var 2) & -0.18 & -0.20 & -0.21 & -0.22 & -0.23 & -0.25 & -0.25 \\
Clustering coefficient of Kin. (Var 3) & 0.12 & 0.22 & 0.29 & 0.31 & 0.30 & 0.24 & 0.24 \\
Clustering coefficient of Nei. (Var 4) & -0.10 & -0.03 & 0.04 & 0.07 & 0.12 & 0.22 & 0.22 \\
Var 1 × Var 3 & 7.06 & 6.47 & 6.10 & 5.9 & 5.80 & 4.77 & 4.77 \\
Var 2 × Var 4 & -0.00 & -0.08 & -0.16 & -0.20 & -0.24 & -0.25 & -0.25 \\
$R^{2}$ & 0.70 & 0.72 & 0.74 & 0.75 & 0.75 & 0.68 & 0.68 \\
\hline
\end{tabular}

\section{Scenario of negative externality effect}

In the scenario of negative externality effect, the converged adoption rate and the speed of convergence are used as response variables to run regression using the Eq. (2). Results are presented in Table 7 (average degree), Table 8 (clustering coefficient), and Table 9 (average path length).

We also found that interaction effects with all the three metrics are significant. Specifically, on the kinship network, average degree undermines the impact of experience effect on the converged adoption rate, but enhances its impact on convergence speed. On the neighbourhood network, the average degree enlarges the impact of externality effect on converged adoption rate, but dilutes its impact on convergence speed. Interaction effects with clustering and distance between households are more prominent. On the kinship network, the interaction effect largely reduces the impact of experience effect on the converged adoption rate and raises it on diffusion speed, thus contributing to a reduced converged adoption rate and helping speed up the convergence of the system. On the neighbourhood network, interaction effect amplifies the impact of externality effect on converged adoption rate and largely reduces that on diffusion speed, which leads the adoption rate to converge to a lower value but at a higher speed. The interaction effect with average path length follows the same pattern but in an opposite direction. The shorter path length is generally associated with lower converged adoption rate and higher

Table 6 Regressions of adoption speed on average path length (positive externality effect)

\begin{tabular}{llllllll}
\hline & Model 1 & Model 2 & Model 3 & Model 4 & Model 5 & Model 6 & Model 7 \\
& $(30 \%)$ & $(40 \%)$ & $(50 \%)$ & $(60 \%)$ & $(70 \%)$ & $(80 \%)$ & $(90 \%)$ \\
\hline (Intercept) & -0.18 & -0.08 & -0.01 & 0.03 & 0.06 & 0.23 & 0.23 \\
Number of households & 0.00 & 0.00 & 0.00 & 0.00 & 0.00 & 0.00 & 0.00 \\
Ratio of seed adopters & 0.90 & 0.84 & 0.81 & 0.80 & 0.79 & 0.57 & 0.57 \\
Experience coefficient $\operatorname{Var} 1)$ & 4.29 & 4.11 & 3.99 & 3.91 & 3.84 & 3.05 & 3.05 \\
Externality threshold $\operatorname{Var} 2)$ & -0.25 & -0.30 & -0.34 & -0.36 & -0.39 & -0.50 & -0.50 \\
APL of Kin. $(\operatorname{Var} 3)$ & 0.01 & -0.01 & -0.02 & -0.02 & -0.02 & -0.02 & -0.02 \\
APL of $\operatorname{Nei}$ (Var 4) & 0.00 & -0.01 & -0.02 & -0.02 & -0.03 & -0.07 & -0.07 \\
Var 1 $\times \operatorname{Var} 3$ & -1.01 & -0.94 & -0.89 & -0.87 & -0.85 & -0.68 & -0.68 \\
$\operatorname{Var} 2 \times \operatorname{Var} 4$ & 0.03 & 0.04 & 0.05 & 0.05 & 0.06 & 0.09 & 0.09 \\
$R^{2}$ & 0.67 & 0.69 & 0.71 & 0.72 & 0.72 & 0.65 & 0.65 \\
\hline
\end{tabular}


Table 7 Regressions of convergence adoption rate and convergence speed on average degree (negative externality effect)

\begin{tabular}{lll} 
& $\begin{array}{l}\text { Model 1 } \\
\text { (Conv. Adp. rate) }\end{array}$ & $\begin{array}{l}\text { Model 2 } \\
\text { (Conv. speed) }\end{array}$ \\
\hline (Intercept) & 0.85 & 0.08 \\
Number of households & 0.00 & 0.00 \\
Ratio of seed adopters & 0.50 & 0.34 \\
Experience coefficient $(\operatorname{Var} 1)$ & 0.50 & 0.90 \\
Externality threshold $(\operatorname{Var} 2)$ & -0.05 & -0.28 \\
Average degree of Kin. $(\operatorname{Var} 3)$ & 0.01 & 0.02 \\
Average degree of Nei. $(\operatorname{Var} 4)$ & 0.01 & -0.01 \\
Var 1 $\times$ Var 3 & -0.03 & 0.02 \\
$\operatorname{Var} 2 \times \operatorname{Var} 4$ & -0.04 & 0.09 \\
$R^{2}$ & 0.11 & 0.74 \\
\hline
\end{tabular}

diffusion speed. Overall, these results suggest that stronger connectivity, higher transitivity, or shorter distance shape the peer effects in a way that the system converges at a lower adoption rate and more speedily.

\section{Conclusions}

This study distinguishes three basic underlying mechanisms through which peer effects in the diffusion of innovation occur: information transmission, experience sharing, and externalities. Correspondingly, peer effects are classified as information effect, experience effect, and externality effect. In the case of diffusion of a rural innovation, we found that each of the three effects played a dominant role at the early, intermediate, and late stages, respectively. Peer effects can be better understood by investigating the specific effects and their roles at different stages of the dynamic diffusion process.

By referring to the diffusion process of a rural innovation in the real world, we developed an agent-based model that incorporates experience and externality effects on a multiplex network. The model allows us to estimate the influence of each specific effect and investigate the interplay of positive and negative effect. In particular, we examined how experience effect and externality effect shape the diffusion jointly. By conducting experiments using the model, we obtained several findings. First, our model successfully replicates the widely acknowledged S-shaped diffusion curve in the scenario of positive

Table 8 Regressions of convergence adoption rate and convergence speed on clustering coefficient (negative externality effect)

\begin{tabular}{lll} 
& $\begin{array}{l}\text { Model 1 } \\
\text { (Conv. Adp. rate) }\end{array}$ & $\begin{array}{l}\text { Model 2 } \\
\text { (Conv. speed) }\end{array}$ \\
\hline (Intercept) & 0.78 & -0.18 \\
Number of households & 0.00 & 0.00 \\
Ratio of seed adopters & 1.02 & 0.24 \\
Experience coefficient $(\operatorname{Var} 1)$ & 0.20 & 1.43 \\
Externality threshold $(\operatorname{Var} 2)$ & -0.05 & -0.28 \\
Clustering coefficient of Kin. $(\operatorname{Var} 3)$ & 0.66 & 0.79 \\
Clustering coefficient of $\operatorname{Nei}(\operatorname{Var} 4)$ & 0.00 & -0.08 \\
Var 1 $\times$ Var 3 & -3.29 & 1.29 \\
$\operatorname{Var} 2 \times \operatorname{Var} 4$ & -0.10 & 0.72 \\
$R^{2}$ & 0.18 & 0.66 \\
\hline
\end{tabular}


Table 9 Regressions of convergence adoption rate and convergence speed on average path length (negative externality effect)

\begin{tabular}{lll}
\hline & $\begin{array}{l}\text { Model 1 } \\
\text { (Conv. Adp. rate) }\end{array}$ & $\begin{array}{l}\text { Model 2 } \\
\text { (Conv. speed) }\end{array}$ \\
\hline (Intercept) & 1.28 & 0.04 \\
Number of households & 0.00 & 0.00 \\
Ratio of seed adopters & -0.98 & 0.97 \\
Experience coefficient $(\operatorname{Var} 1)$ & -0.44 & 2.87 \\
Externality threshold $(\operatorname{Var} 2)$ & -0.05 & -0.28 \\
APL. of Kin. $(\operatorname{Var} 3)$ & -0.10 & -0.10 \\
APL. of Nei. $(\operatorname{Var} 4)$ & -0.04 & 0.08 \\
$\operatorname{Var} 1 \times \operatorname{Var} 3$ & 0.52 & -0.18 \\
$\operatorname{Var} 2 \times \operatorname{Var} 4$ & 0.28 & -0.59 \\
$R^{2}$ & 0.22 & 0.63 \\
\hline
\end{tabular}

externality effect. This finding is consistent with the pattern argued in the theory of diffusion of innovations (Rogers 2003). However, when there is a negative effect in the system (the negative externality effect, in our case), the diffusion will vibrate around a middlelevel rate between 0 and 100\%. Accordingly, the diffusion curve will be a fluctuating one. This curve demonstrates the trajectory of the interplay of opposite effects. In reality, many innovations do not end up being adopted by the whole population of potential adopters. This could be accounted for by the existence of negative effects. However, the role of negative effects is usually left undiscussed in literature.

Second, our simulation results show that experience effect has a relatively higher influence on diffusion at the earlier stage, whereas externality effect dominates at the later stage in the scenario of positive externality effect. Along with the findings regarding the information effect we learnt in the real-world case, it is likely to be true that each of the three effects plays a dominant role at a different stage of a complete diffusion process, one after another. This pattern is not found in the scenario of negative externality effect, although both influences are still significant. This is perhaps due to the fact that the opposite effects can offset each other in the diffusion process, which leaves the feature associated with the strength of the effects inconspicuous. In general, the result suggests that a diffusion process should be examined from a dynamic perspective, and the influence of each specific peer effect needs to be estimated by the period in the diffusion process. Although a couple of attempts to distinguishing mechanisms underlying peer effects can be found (Banerjee et al. 2013; Bursztyn et al. 2014), none of them examines the mechanisms at different periods of the diffusion process.

Third, we found that network properties of connectivity, transitivity, and network distance can indirectly influence diffusion through the specific peer effect that occurs on the network. Specifically, in the scenario of positive externality effect, both experience effect and externality effect are generally enhanced in networks that are well connected, highly transitive, and with low distance between individuals. The effectiveness of the diffusion is thus improved. These findings are in line with existing studies (Janssen and Jager 2003; Laciana et al. 2013; Peres 2014). However, we find no studies considering the negative effect. In the case of negative effect, our work shows that these characteristics shape the two effects in a way that helps speed up diffusion, but tends to lower the coverage it 
could reach. These together suggest that each specific peer effect is reshaped by the structure of the network where it occurs. This could further influence the effectiveness of the diffusion.

Overall, our study shows that it needs to delve into the specific underlying causal mechanisms when we study peer effects. The influence of each underlying mechanism varies by the stages of the diffusion process and by the topological characteristics of the underlying network. In addition, the negative effect, if exists, should be taken into account. It can substantially modify how the diffusion proceeds and its outcome. Our study also suggests that it could be an effective approach to investigate specific peer effects by conducting simulation on social networks.

\section{Endnotes}

${ }^{1}$ Refer to Xiong et al. (2016) for a detailed survey.

${ }^{2}$ This is what is found in the empirical study. Refer to Xiong and Payne (2017) for a detailed discussion.

${ }^{3}$ The density at the 20 th round is as high as over $15 \%$ because it also contains the cases that take more than 20 rounds to converge and those do not converges to full adoption.

${ }^{4}$ To have a better visualisation, we average the adoption rates over repetitive runs. The speed at which the adoption rate grows varies significantly over different settings, so a fat midsection of the integrated graph is observed when plotting all the curves in one frame of axes.

${ }^{5}$ To measure the number of rounds over all runs with a unified standard, we split the values of adoption rate into 10 diffusion bins. The values higher than $10 \%$ and less than $20 \%$ are in the $10 \%$ bin, those higher than $20 \%$ and less than $30 \%$ are in the $20 \%$ bin, and so on, up to the $90 \%$ bin. For instance, it takes 4 rounds for the diffusion to first achieve the diffusion level of, say, $58 \%$. The actual adoption rate at the 4 th round is $58 \%$. Suppose the seed ratio is $10 \%$, then the growth rate for the system to achieve $50 \%$ diffusion in this setting is $(58 \%-10 \%) / 4=12 \%$. There are cases that the diffusion speed is the same for several diffusion bins. For instance, suppose the adoption rate jumps from 34 to $66 \%$ at the fourth round, and the seed ratio is $10 \%$, the diffusion speed for the $30 \%$ bin is thus $(34 \%-10 \%) / 3=8 \%$, and that for the $40 \%, 50 \%$, and $60 \%$ bins are all $(66 \%-10 \%) / 4=14 \%$.

${ }^{6}$ All effect sizes reported in the table were significantly larger than the minimally meaningful effect of 0.1 standard deviations. This is the same for all the following tables that report regression results.

${ }^{7}$ The quality of fit for the models using convergence adoption rate as the response variable (model 1 in Table 2, 7, 8, and 9) is low. Other factors or non-linear structures could provide a better model fit. However, in here, we have focused on specific models, and the exploration of the best fitted models is beyond the scope of the study.

Acknowledgements

This work was supported by the key project of scientific research sponsorship programme of Hubei Province, China: Governance on Farmers' Pesticide Use Behaviour: A Social Network Analysis Perspective (ID: D20151706).

Authors' contributions

$\mathrm{HX}$ led the writing of the manuscript with all other authors contributing to ideas and writing. All authors read and approved the final manuscript. 


\section{Publisher's Note}

Springer Nature remains neutral with regard to jurisdictional claims in published maps and institutional affiliations.

\section{Author details}

${ }^{1}$ Agricultural Economics and Policy Group, ETH Zurich, 8092 Zurich, Switzerland. ${ }^{2}$ School of Economics and Management, Wuhan Polytechnic University, Wuhan, 430048 China. ${ }^{3}$ Center for Data Science, RTI International, Durham, 27709-2194 USA.

Received: 14 September 2017 Accepted: 21 February 2018

Published online: 07 March 2018

\section{References}

Alcalde, P (2013). A behavioral model of non-linear peer effects in cognitive achievement. PhD thesis: University of Pennsylvania. http://repository.upenn.edu/dissertations/AAI3565014. Accessed 24 Feb 2018.

Arenas, A, BarthelemyY, M, Gleeson, JP, Moreno, Y, Porter, MA (2014). Multilayer networks. Journal of Complex Networks, 2, 203-271.

Bandiera, O, \& Rasul, I (2006). Social networks and technology adoption in northern Mozambique. The Economic Journal, 116(514), 869-902.

Banerjee, A, Chandrasekhar, AG, Duflo, E, Jackson, MO (2013). The diffusion of microfinance. Science, 341(6144), 1236498-1-1236498-7.

Bramoulle, Y, Djebbari, H, Fortin, B (2009). Identification of peer effects through social networks. Journal of econometrics, 150(1), 41-55.

Bursztyn, L, Ederer, F, Ferman, B, Yuchtman, N (2014). Understanding mechanisms underlying peer effects: evidence from a field experiment on financial decisions. Econometrica, 82(4), 1273-1301.

Carrell, SE, Sacerdote, BI, West, JE (2013). From natural variation to optimal policy? The Lucas critique meets peer effects. Econometrica, 81(3), 855-882.

Conley, TG, \& Udry, CR (2010). Learning about a new technology: Pineapple in Ghana. The American Economic Review, 100(1), 35-69.

Dahl, GB, Lken, KV, Mogstad, M (2014). Peer effects in program participation. American Economic Review, 104(7), 2049-74.

Deffuant, G, Huet, S, Amblard, F (2005). An individual-based model of innovation diffusion mixing social value and individual benefit. American Journal of Sociology, 110(4), 1041-1069.

Erdős, P, \& Rényi, A (1959). On random graphs. Publicationes Mathematicae (Debrecen), 6, $290-297$.

Goldenberg, J, Libai, B, Muller, E (2001). Talk of the network: a complex systems look at the underlying process of word-of-mouth. Marketing Letters, 12(3), 211-223.

Goldsmith-Pinkham, P, \& Imbens, GW (2013). Social networks and the identification of peer effects. Journal of Business \& Economic Statistics, 31(3), 253-264

Goolsbee, A, \& Klenow, PJ (1999). Evidence on learning and network externalities in the diffusion of home computers. National bureau of economic research. Technical report.

Granovetter, M (1978). Threshold models of collective behavior. American Journal of Sociology, 83(6), 1420.

Guryan, J, Jacob, B, Klopfer, E, Groff, J (2008). Using technology to explore social networks and mechanisms underlying peer effects in classrooms. Developmental Psychology, 44(2), 355

Heard, D, Bobashev, GV, Morris, RJ (2014). Reducing the complexity of an agent-based local heroin market model. PloS ONE, 9(7), 102263.

Janssen, MA, \& Jager, W (2003). Simulating market dynamics: interactions between consumer psychology and social networks. Artificial Life, 9(4), 343-356.

Kiesling, E, Günther, M, Stummer, C, Wakolbinger, LM (2012). Agent-based simulation of innovation diffusion: a review. Central European Journal of Operations Research, 20(2), 183-230.

Kremer, M, \& Levy, D (2008). Peer effects and alcohol use among college students. The Journal of Economic Perspectives, 22(3), 189-189.

Laciana, CE, Rovere, SL, Podest, GP (2013). Exploring associations between micro-level models of innovation diffusion and emerging macro-level adoption patterns. Physica A: Statistical Mechanics and its Applications, 392(8), 1873-1884.

Luan, YJ, \& Neslin, S (2009). The development and impact of consumer word of mouth in new product diffusion. Tuck School of Business Working Paper No. 2009-65. https://doi.org/10.2139/ssrn.1462336. Accessed 24 Feb 2018.

Munshi, K (2004). Social learning in a heterogeneous population: technology diffusion in the Indian Green Revolution. Journal of Development Economics, 73(1), 185-213.

Peres, R (2014). The impact of network characteristics on the diffusion of innovations. Physica A: Statistical Mechanics and its Applications, 402(0), 330-343.

Rogers, EM (2003). Diffusion of innovations, 5th edn. New York: Free Press.

Singh, P, Sreenivasan, S, Szymanski, BK, Korniss, G (2013). Threshold-limited spreading in social networks with multiple initiators. Science Report, 3(2330).

Sorensen, AT (2006). Social learning and health plan choice. RAND Journal of Economics, 37(4), 929-945

Watts, DJ, \& Strogatz, SH (1998). Nature, 393(6684), 440-442.

Wellek, S (2010). Testing statistical hypotheses of equivalence and noninferiority, 2nd edn. New York: CRC Press.

Xiong, H, Paynea, D, Kinsellac, S (2016). Peer effects in the diffusion of innovations: theory and simulation. Journal of Behavioral and Experimental Economics, 63, 1-1713.

Xiong, H, \& Payne, D (2017). Characteristics of Chinese rural network: evidence from villages in central China. Chinese Journal of Sociology, 1(1), 74-1797. 